$$
\begin{array}{cc}
\text { Berechnet } & \text { Gebraucht } \\
\text { cem Natronlange } 3.8 & 3.8
\end{array}
$$

Phenylazoxazolcarbonsäure giebt mit Kupferacetat und Bleiacetat weder eine Fällung noch eine Färbung, mit Silbernitrat und Mercurichlorid dagegen einen weissen Niederschlag und durch Behandlung des Silbersalzes mit Jodmethyl den bei $35^{\circ}$ schmelzenden Methyläther. Mit Acetylchlorid und Essigsäureanhydrid liefert sie keine Acetylverbindung, welche Indifferenz der Abwesenheit von Oximhydroxylen gemäss der Formel entspricht. Charakteristisch ist auch die grosse Beständigkeit dieser Azoxazolsäure gegenüber Säuren; denn sogar längere Berührung mit concentrirter Schwefelsäure vermag sie nicht zu verändern. Versuche, aus ihr das von Russanow ${ }^{1}$ ) schon dargestellte Phenylazoxazol zu erhalten, blieben obne Erfolg. Die Destillation des Silbersalzes konnte wegen seiner Explosibilität nicht ausgefübrt werden.

311. A. Hantzsch: Ueber Beziehungen zwischen Constitution, Configuration und chemischem Verhalten der Oxime.

(Eingegangen am 22. Juni; mitgetheilt in der Sitzung von Hrn. H. Jahn.)

Dass die Natur und die Verkettung der Atome innerhalb des Moleküles, wie sie durch die Constitutionsformeln der Structurchemie ausgedrückt wird, auf ihre relative räumlicbe Anordnung, also auf die Configuration einen sehr bedeuteuden Einfluss ausübt, ist besonders für stereoisomere Koblenstoffierbindungen bereits ron verschiedenen Seiten hervorgeboben and in einigen Fällen auch im Detail verfolgt worden. Die Configuration wird bekanntlich beeinflusst durch den besonders von $J$. Wislicenus betonten elektrischen Gegensatz zwischen positiven und negativen Atomen bezw. Atomgruppen, der sich als "Anziehung bezw. Abstossung " äusse it, danach ist z. B. Fumarsäure begänstigter ist als Maleïnsäure, weil die ungleichartige Radicale $\mathrm{CO} \overline{\mathrm{O}} \mathrm{H}$ und $\hat{\mathrm{H}}$ einander mebr anzieben, als die gleichartigen; ebenso wird die Nichtexistenz zweier Dichlorätbylene z. B. darauf zurückgeführt, dass die Configuration $\begin{aligned} & \mathrm{Cl} . \mathrm{C} \cdot \mathrm{H} \\ & \mathrm{Cl} . \ddot{\mathrm{C}} . \mathrm{H}\end{aligned}$ isomeren $\begin{aligned} & \mathrm{H} \cdot \mathrm{C} \cdot \mathrm{Cl} \\ & \mathrm{Cl} . \ddot{\mathrm{C}} \cdot \mathrm{H}\end{aligned}$ in Folge der starken Anziehung zwischen Chlor 
und Wasserstoff so unbegünstigt ist, dass sie bisher nicht gefasst werden konnte, sondern sich spontan in diese letztere umlagert. Dieser auf die Natur der Radicale zurückgeführte, gewissermaassen rein chemische Einfluss der das Molekül zusammensetzenden Atome auf dessen răumliche Anordnung ist aber bekanntlich nicht ïberall direct zu erkennen; er wird jedenfalls durch ein zweites Moment sehr wesentlich modificirt, welches etwa gleichzeitig von V. Meyer und A uwers, sowie ron C. Bischoff bei Untersuchung gewisser Dicarbonsäuren und Ketonsäuren hervorgehoben wurde, und welches man wohl schwerlich auf eine andere Ursache als auf die im einzelnen freilich noch völlig unbekannten räumlichen Dimensionen der Atome bezw. Atomgruppen zurückführen kann. Man wird geradeza von einer $\gg$ Platzfragec der Atome innerbalb des Moleküles ${ }^{1)}$ reden dürfen; gewisse Configurationen werden dèshalb unbegünstigt sein, oder überhaupt nicht bestehen können, ja, gewisse der Structurformel nach sehr wohl mögliche Reactionen werden deshalb nicht eintreten können, weil die betreffenden Gruppen nicht neben einander Platz finden, bezw. sich nicht so sebr nähern können, dass sie in ihre gegenseitige Wirkungssphäre gelangen. Um vorläufig nur einige Beispiele für den ersten Fall zu geben, so besteben bekanntlich zwar die Säuren (CH.COOH) $)_{2}$ als Fumar- und Maleïnsäure in beiden Configurationen und $\mathbf{z w a r}$ letztere als unbegünstigte Form, ihre Dimethylderivate $\left(\mathrm{CCH}_{3} . \mathrm{COOH}\right)_{2}$ dagegen nur in einer einzigen Configuration und zwar in derselben malë̈noiden) Anordnung, welche bei den ursprünglichen Säuren labil ist; so bestehen bekanntlich $\mathrm{zwar}$ die beiden Benzaldoxime $\mathrm{C}_{6} \mathrm{H}_{5}$. CNOH . H; ersetzt man aber, wie in dem erwähnten Falle, das an Kohlenstoff gebundene Wasserstoffatom durch Methyl, so existirt das so resultirende Oxim des Acetophenons $\mathrm{C}_{6} \mathrm{H}_{5}$. CNOH. $\mathrm{CH}_{3}$ nur in einer einzigen Configuration. Diese leicht zu vermehrenden Beispiele zeigen also, dass durch Vertretung von Wasserstoff durch Methyl die Möglichkeiten der räumlichen Anordnung verändert, bezw. vermindert werden, dass die zwar denkbare zweite Configuration mehr oder minder labil, bezw. gar nicht mehr existenzfähig wird. Diese Erscheinungen können nicht, oder wenigstens nicht direct und unmittelbar auf den Gegensatz zwischen positiv und negativ zurückgeführt werden; denn da Methyl für positiver angenommen wird, als Wasserstoff, so sollte eigentlich, ebenso wie die nicht existirende Dimethylfumarsäure $\begin{array}{r}\mathrm{CH}_{3} \cdot \mathrm{C} \cdot \mathrm{COOH} \\ \mathrm{COOH} \cdot \ddot{\mathrm{C}} \cdot \mathrm{CH}_{3}\end{array}$ stabilersein

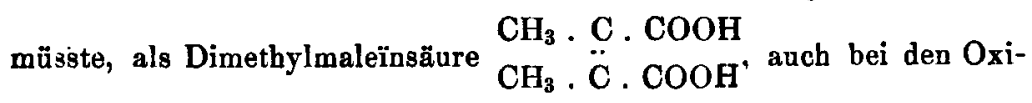

1) vergl. z. B. C. Bisch off, Jahrbuch d. Chemie für 1891, Seite 135 ff 177 etc. 


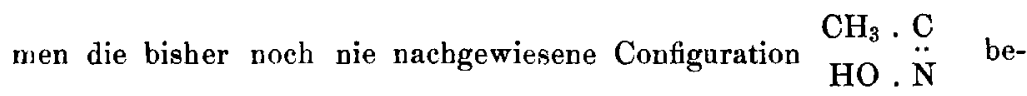
günstigter sein, als die Configuration $\begin{array}{r}\text { H. } \\ \text { HO } . \ddot{N}\end{array}$. Wenn gerade das Umgekehrte eintritt, so wird man sich eben vorläufig lieber damit begnügen, den dem Wasserstoff entgegengesetzten Einfluss der Methylgruppe auf die Configuration einfach als eine Folge der grösseren oder geringeren Anziehung der Gruppen aufeinander zu bezeichnen - ohne dass damit wirklich anziehende oder gar abstossende Kräfte angenommen oder überhaupt bestimmte Vorstellungen von der wahren Ursache derartiger Erscheinungen erweckt werden sollen. Ebenso wenig soll auch behauptet werden, dass der elektrische Gegensatz der Gruppen innerhalb des Moleküles bei stereoisomeren Oximen keinen Einfluss auf die Stabilitätsverhältnisse der Configurationen habe. Im Gegentheile lässt sich derselbe gerade hier bei einfacheren Oximen in dem bekannten Sinne nacbweisen: es hat sich $z$. B. aus dem elektrischen Leitvermögen von Oximidosäuren die von vornherein wahrscheinlichere Thatsache bestätigt, dass von den Bestandtheilen der Oximgruppe $\mathrm{N} . \mathrm{OH}$ der Stickstoff negativer ist als der Wasserrest. Da nun bei den bisher untersuchten Oximen der Form $\mathrm{CH}_{3}$. $\mathrm{CNOH}$. X das Methyl seinerseits stets positiver ist als das mit $\mathrm{X}$ bezeichnete Radical, so werden gemäss der Erfahrung die Beziehungen herrschen:

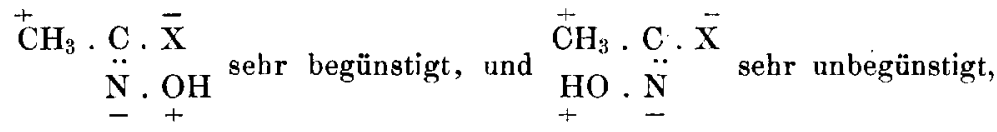

desgleichen bei den Oximen $\mathrm{C}_{6} \mathrm{H}_{5}$. CNOH. COOH, in welchen $\mathrm{COOH}$ negativer ist als $\mathrm{C}_{6} \mathrm{H}_{5}$, die thatsächlich bestätigten Beziehungen:

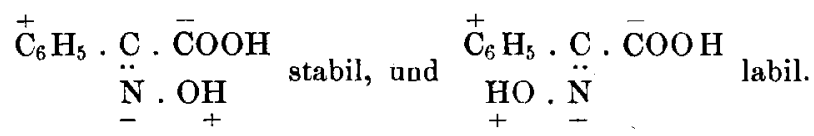

Indessen lässt sich diese einfache Abhängigkeit der Configuration von der elektrischen Wirkung der Radicale schon bei etwas complicirteren Oximen, besonders bei solchen mit längerer Kohlenstoffkette, nicht mehr ungetrübt erkennen; dies dürfte vielleicht nur in der Unzulänglichkeit unserer Stereoformeln beruhen, welche ja bisher immer nur ein einseitiges und ganz unvollständiges Bild der intramolecularen Lagerungsverhältnisse geben. In Wirklichkeit könnten gerade bei complicirteren Verbindungen gewisse Gruppen ganz andere Entfernungen aufweisen, als es nach derartigen Annäherungsformeln den Anschein hat. Ich glaube daher, um es zu wiederbolen, dass der Einfluss der Radicale auf die Ċonfiguration vorläufig viel eher ver- 
schleiert, als geklärt wird, wenn man diesen Einfluss direct auf elektrischen Gegensatz zurückzuführen sich bestrebt, als wenn mau sich mit der Thatsache des specifischen Einflusses gewisser Gruppen begnügt und denselben im Einzeluen möglichst genau untersucht. Jedenfalls würde es auch auf dem Gebiete der Oxime zu Trugschlüssen führen, wenn man ans der elektrischen Anziehung bezw. Abstossung der Radicale a priori Stabilität und Labilität der Configuration bestimmen wollte.

Der Einfluss der Constitution anf die Configuration, d. i. der Einfluss der Radicale auf die Existenz, Beständigkeit und die Reactionen stereoisomerer Moleküle ist nun wohl anf keinem Gebiete im Einzelnen bereits so genau ermittelt worden, als innerhalb der an sich kleinen Gruppe der Oxime. Diese Ergebnisse sind in zahlreichen Einzeluntersuchungen verstreut, und z. Th. nur versteckt enthalten; es dürfte aber gegenwärtig an der Zeit sein, dieselben im Zasammenhange vorzuführen und die sich ergebenden Regelmässigkeiten hervorzuheben.

I. Einfluss der Constitution asymmetrischer Oxime auf die Beständigkeit bezw. Existenz der beiden Configurationen

$$
\begin{array}{rl}
\mathrm{X} \cdot \mathrm{C} \cdot \mathrm{Y} & \mathrm{X} \cdot \mathrm{C} \cdot \mathrm{Y} \\
\mathrm{HO} \cdot \mathrm{N} & \text { und } \\
\ddot{\mathrm{N}} \cdot \mathrm{OH}
\end{array}
$$

Dass die Existenz bezw. Beständigkeit der beiden Configurationen in bohem Grade von der Natur der beiden mit $\mathrm{X}$ und $\mathrm{Y}$ bezeicbneten Radicale eiues asymmetrischen Oxims abhängt, ist beinahe gleichzeitig mit Aufstellung der neuen Theorie der Oxime von Werner und mir erkannt worden. Aber seitdem sind beim genaueren Studium der verschiedensten Oxime auch feinere qualitative Unterschiede dieser Art bekannt geworden; es sind zahlreiche Anbaltspunkte dufür gefunden

1) Ueber diesen von versebiedenen Autoren undauch von mir selbst gebrauchten Ausdruck der »Abstossung « zweier Radicale von einander (z. B. des Methyls vom Oximhydroxyl) sei zur Vermeidung allfälliger Missverständnisse Folgendes bemerkt: Eine derartige Ausdrucksweise bedeutet nach meiner Meinung nicht, dass sich die betr. Radicale (z. B. Methyl und Hydroxyl in den Oximen von der Configuration $\left.\begin{array}{r}\mathrm{CH}_{3} . \mathrm{C} . \mathrm{X} \\ \ddot{\mathrm{N}} . \mathrm{OH}\end{array}\right)$ wirklich abstossen; im Gegentheile werden auch sie eine gewisse Anziehung auf einander ausüben; allein dieselbe ist geringer, als die ihr entgegenwirkenden Kräfte (z. B. als die Wirkung des Radicales $X$ auf das Oximhydroxyl), und kommt deshalb nio bezw. kaum zur Wirkung, so dass man kürzer und prăgnanter, obgleich weniger correct, in diesen Fällen von »Abstossung« sprechen kann. 
worden, dass diese Radicale je nach ihrer Natur nicht nur versehieden, sondern immer in demselben Sinne verschieden wirken. Man kann aus dem Vergleich der verschiedensten Oxime geradezu eine $\mathbf{S k a l a}$ der Wirksamkeit der Radicale $X$ und $Y$ hinsichtlich ihrer Anziehung auf das Hydroxyl aufstellen und danach die Beständigkeit bezw. Existenzfähigkeit der beiden Stereoisomeren aus dem rereinten Einflusse dieser beiden Radicale herleiten.

Diese Scala, welche allerdings nur die häufigst rorkommenden Radicale umfasst, und als erster derartiger Versuch nur auf annähernde Genauigkeit Anspruch macht, beginnt mit den das Oximhydroxyl am stärksten anziehenden und schliesst mit den am schwächsten anziehenden Radicalen, so dass jedes von links nach rechts folgende Radical weniger intensiv wirkt, als das vorangehende:
1. $\mathrm{COOH} . \mathrm{CH}_{2}$
2. $\mathrm{COOH} \cdot \mathrm{CH}_{2} \cdot \mathrm{CH}_{2}$
3. $\mathrm{COOH}$ 4. $\mathrm{C}_{6} \mathrm{H}_{5}$
5. $\mathrm{C}_{6} \mathrm{H}_{4} \mathrm{X}$ (m od. $\left.p\right)$
6. $\mathrm{C}_{6} \mathrm{H}_{5}, \mathrm{CO}$
7. $\mathrm{C}_{6} \mathrm{H}_{4} \mathrm{X}$ (ortho)
8. $\mathrm{C}_{4} \mathrm{H}_{3} \mathrm{~S}\left(\mathrm{C}_{4} \mathrm{H}_{3} \mathrm{O}\right)$
9. $\mathrm{C}_{\mathrm{n}} \mathrm{H}_{2 \mathrm{n}+1}$
10. $\mathrm{CH}_{3}$.

Danach besteht also der schärfste Gegensatz zwischen dem das Hydroxyl am stärksten "abstossenden \& Methyl und dem es am stärksten anziehenden carboxylirten Methyl $\left(\mathrm{CH}_{2}, \mathrm{COOH}\right)$. Einige einfache und zum Theil nicht unwichtige Radicale, wie $\mathrm{Cl}, \mathrm{OC}_{2} \mathrm{H}_{5}$, $\mathrm{CN}$ u. a. sind in obige Reihe nicht aufgenommen, weil ihre Wirksamkeit nur an einigen Oximen beobachtet und noch nicht genügend festgestellt ist Vor allem ist aber vorlüufig unberücksichtigt geblieben der Wassersteff, indess ans einem anderen Grunde; denn die entsprechenden Wasserstoff rerbindungen, die Aldoxime H. CNOH.Y, sind sehr gut studirt; allein ihre Configuration wechselt in scheinbar so unregelmässiger Weise, dass man dem Wasserstoff auch hier wie in vielen anderen Fällen eine Ausnabmestellung zuertheilen muss; in der Regel würde er an die Spitze der gesammten Reihe zu stehen kommen; bisweilen wäre er in der Mitte, in der Nähe des Phenyls einzureiben; er wird daher lieber hier ausgeschaltet und am Schlusse besonders betrachtet werden.

Der graduell verschiedene Einfluss der Radicale auf die Beständigkeit der beiden Configurationen lässt sich am besten, natürlich immerhin nur qualitativ, verfolgen, wenn man in einem Molekül von der Form X.CNOH. $Y$ das $X$ constant lässt und das $Y$ variirt. Hierfür eignet sich besonders der Specialfall $X=\mathrm{C}_{6} \mathrm{H}_{5}$, weil dieses Radical eine mittlere Stellang einnimmt and die Verbindungen der allgemeinen Structurformel $\mathrm{C}_{6} \mathrm{H}_{5} . \mathrm{CNOH} . \mathrm{Y}$ besonders vollständig bekannt sind. 
$\mathrm{Oxime} \mathrm{C}_{6} \mathrm{H}_{5}$. CNOH . $\mathrm{Y}$

1. $\mathrm{Y}=\mathrm{CH}_{2}$. $\mathrm{COOH} \quad \mathrm{C}_{6} \mathrm{H}_{5}$. CNOH. $\mathrm{CH}_{2}$. $\mathrm{COOH}$, Phenylketoximessigsäuren (Oxime der Benzoylessigsäure).
a) $\mathrm{C}_{6} \mathrm{H}_{5} \cdot \mathrm{C} \cdot \mathrm{CH}_{2} \cdot \mathrm{COOH}$
$\mathrm{N} . \mathrm{OH}$
b) $\mathrm{C}_{6} \mathrm{H}_{5} \cdot \underset{.}{\mathrm{C}} \cdot \mathrm{CH}_{2} \cdot \mathrm{COOH}$ HO..$\ddot{N}$

Phenylsynketoximessigsăure, einzig Phenylantiketoximessigsäure, nnbebelsannt, im freien Zustande über- kannt, aber in der Lösung des Oxims hauptnur alsinneresAnhydrid (Phenyl- in concentrirter Schwefelsäure. vorsynoxazolon) beständig. übergehend anzunehmen ').

2. $\mathrm{Y}=\mathrm{CH}_{2} \cdot \mathrm{CH}_{2} \cdot \mathrm{COOH} \mathrm{C}_{6} \mathrm{H}_{6}, \mathrm{CNOH}, \mathrm{CH}_{2}, \mathrm{CH}_{2} \cdot \mathrm{COOH}$, Phenylketoximpropionsăuren (Oxime der Benzoylpropionsäure).

a) $\mathrm{C}_{6} \mathrm{H}_{5} \cdot \mathrm{C} \cdot \mathrm{CH}_{2} \cdot \mathrm{CH}_{2} \cdot \mathrm{COOH}$ $\ddot{\mathrm{N}} . \mathrm{OH}$

Phenylsynketoximpropionsăure, änsserst stabil, aber nicht als inneres Anhydrid zu erhalten. b) $\mathrm{C}_{6} \mathrm{H}_{5} \cdot \mathrm{C} \cdot \mathrm{CH}_{2} \cdot \mathrm{CH}_{2} \cdot \mathrm{COOH}$ HO. $\ddot{\mathbf{N}}$

Phenylantiketoximpropionsäure, ausserst labil, aus dem stabilen Oxim durch concentrirte Schwefelsäure entstehend und im freiem Zustande rasch in jenes zurückgehend ${ }^{2}$ ).

\section{3. $\mathrm{Y}=\mathrm{COOH} \quad \mathrm{C}_{6} \mathrm{H}_{5}$. CNOH.COOH,}

Phenylketoximcarbonsäuren (Oxime der Phenylglyoxylsäure).
a) $\mathrm{C}_{6} \mathrm{H}_{5} \cdot \mathrm{C} \cdot \mathrm{COOH}$
$\ddot{\mathrm{N}} \cdot \mathrm{OH}$
b) $\mathrm{C}_{6} \mathrm{H}_{5} \cdot . . \mathrm{C} . \mathrm{COOH}$
HO.

$$
\text { HO.N }
$$

Phenylsynketoximcarbonsäure, stabil, aber durch gewisse Agenzien, d. i. in Form gewisser Derivate, in die labile

Form übergehend.

Phenylantiketoximcarbonsäure, labil, in freiem Zustande zum Theil langsam in das stabile Isomere freiwillig über. gehend, völlig durch Säuren ${ }^{3}$ ).
4. $\mathrm{Y}=\mathrm{C}_{6} \mathrm{H}_{4} \mathrm{X}(p$ od. $m)$
$\mathrm{C}_{6} \mathrm{H}_{5}$. CNOH . $\mathrm{C}_{6} \mathrm{H}_{4} \mathrm{X}$, $p$-bezw. $m$-substituirte Benzophenonoxime.

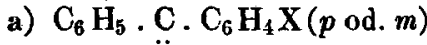 N. $\mathrm{OH}$

im Allgemeinen labil und durch Sãuren in die stabilen Oxime übergehend.
b) $\mathrm{C}_{6} \mathrm{H}_{5} \cdot \mathrm{C} \cdot \mathrm{C}_{6} \mathrm{H}_{4} \mathrm{X}(p$ od. $m)$ HO . N im Allgemeinen stabil.

(Stabilităt und Labilitit werden hier wiederum von der Natur des Substituenten ganz äbnlich beeinflusst, wie nach der obigen Scala ${ }^{4}$ ).

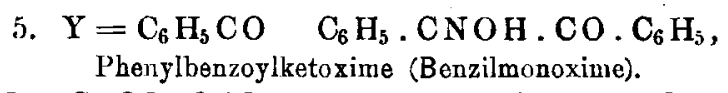
a) $\mathrm{C}_{6} \mathrm{H}_{5} \cdot \mathrm{C} \cdot \mathrm{CO} \cdot \mathrm{C}_{6} \mathrm{H}_{5}$

$$
\ddot{\mathrm{N}} \text {. OH }
$$
b) $\mathrm{C}_{6} \mathrm{H}_{5}, \mathrm{C} \cdot \mathrm{CO} \cdot \mathrm{C}_{6} \mathrm{H}_{5}$ HO. N

Phenylsynbenzoylketoxim, labil.

Phenylantibenzoyllketoxim, stabil ${ }^{5}$ ).

1) A. Hantzseh, vergl. diese Berichte XXIV, 496.

2) W. Dollfus, diese Berichte $X X V, 1933$.

3) A. Hantzsch, diese Berichte XXIV, 41.

4) V. Meyer, diese Berichte XXIV, $4025-4058$.

5) A. W. Smith, diese Berichte XXII, 537.

Bericlate d. D. chem. Gesellschaft. Jahrg. XXV. 


\section{6. $\mathrm{Y}=\mathrm{C}_{6} \mathrm{H}_{4} \mathrm{X}(\mathrm{o}) \quad \mathrm{C}_{6} \mathrm{H}_{5} . \mathrm{CNOH} . \mathrm{C}_{6} \mathrm{H}_{4} \mathrm{X}(\mathrm{o})$,}

Ortho-substituirte (bezw. methylirte) Benzophenonoxime.

Vorlänfig nur für $\mathrm{X}=\mathrm{CH}_{3}$, d. i. für die Oxime des $o$-Tolylphenylketons and Xylylphenylketons untersucht; dort ist:
a) $\mathrm{C}_{6} \mathrm{H}_{5} \cdot \mathrm{C} \cdot \mathrm{C}_{6} \mathrm{H}_{4} \mathrm{XCH}_{3}(\mathrm{o})$
$\ddot{N}$. OH
sehr labil, da es sich bereits durch
b) $\mathrm{C}_{6} \mathrm{H}_{5} \cdot \mathrm{C} \cdot \mathrm{C}_{6} \mathrm{H}_{4} \mathrm{XCH}_{3}(\mathrm{o})$
HO.N
sehr stabil ${ }^{1}$.

Essigsăure langsam umlagert.

$$
\text { 7. } \mathrm{Y}=\mathrm{CH}_{3}\left(\mathrm{C}_{\mathrm{n}} \mathrm{H}_{2 \mathrm{n}+1}\right) \quad \mathrm{C}_{6} \mathrm{H}_{5} . \mathrm{CNOH} . \mathrm{CH}_{3} \text {, }
$$

Phenylmethylketoxime (Oxime des Acetophenons).
a) $\mathrm{C}_{6} \mathrm{H}_{5} \cdot \mathrm{C} \cdot \mathrm{CH}_{3}$
N. OH
b) $\mathrm{C}_{6} \mathrm{H}_{5} \cdot \mathrm{C} \cdot \mathrm{CH}_{8}$
HO. $\ddot{N}$

Phenylsynmethylketoxim, unbekannt. Phenylantimethylketoxim, einzig bekannt.

Die Beständigkeit der beiden Conflgurationen für die Oxime von der allgemeinen Structurformel $\mathrm{C}_{6} \mathrm{H}_{5}$. CNOH. $\mathrm{Y}$ verschiebt sich also nach der Natur des Radicales $X$, bezw. nach seiner Stellung im Sinne der obigen Scala: Gegenüber dem stärkst anziehenden Radical $\mathrm{COOH} . \mathrm{CH}_{2}$ vermag sich das Phenyl gar nicht geltend zu machen; die Configuration mit Nachbarstellung von Phenyl und Hydroxyl fehlt; die Gruppe $\mathrm{COOH} . \mathrm{CH}_{2} \cdot \mathrm{CH}_{2}$ wirkt bereits etwas schwächer; die Configuration mit Nachbarschaft von Phenyl und Hydroxyl vermag wenigstens unter gewissen Bedingungen zu existiren, wenngleich sie äusserst labil ist; die Gruppe $\mathrm{COOH}$ wirkt ebenfalls noch stärker als Phenyl, aber das Oxim mit Nachbarschaft von Phenyl und Hydroxyl ist doch bereits haltbar, wennschon immer noch labil. Nunmehr würde gegen $X=\mathrm{C}_{6} \mathrm{H}_{5}$ für $\mathrm{Y}$ der Wasserstoff folgen; denn die beiden Benzaldoxime $\mathrm{C}_{6} \mathrm{H}_{5}$. CNOH . $\mathrm{H}$ sind annähernd von gleicher Beständigkeit, Wasserstoff und Benzolrest also von annähernd gleicher Wirksamkeit (die Labilität des Benzsynaldoxims besteht nur gegenüber verdünnten Säuren). Jetzt aber kehren sich die Verhältnisse um: gegenüber den nun folgenden Gruppen gewinnt das Phenyl immer mehr die Oberhand; para- und meta-substituirte Benzophenonoxime sind, mit einziger Ausnahme des $p$-Methoxylderivates (Anisylphenylketoxims), stets etwas stabiler in der Configuration mit Nachbarstellung von Phenyl und Hydroxyl; dies steigert sich bei den_orthosubstituirten Oximen, von denen allerdings nur Methylderirate bisher in dieser Richtung untersucht sind. In diesen Oximen, bezw. der Gruppe $\mathrm{C}_{6} \mathrm{H}_{4} \cdot \mathrm{CH}_{3}$ gegenüber wirkt das Benzoyl $\mathrm{C}_{6} \mathrm{H}_{5}$. $\mathrm{CO}$ noch etwas stärker anziehend und hat deshalb in der obigen Reihenfolge seinen Platz vor dem orthosubstituirten Benzolrest erhalten - wobei indess nicht ge-

1) A. W. Smith, diese Berichte XXIV, 4057. 
sagt sein soll, dass bei besonderer Beschaffenheit des Orthosubstituenten (X) nicht auch gewisse Radicale der Formel $\mathrm{C}_{6} \mathrm{H}_{4} \mathrm{X}(0)$ vor dem Benzoyl rangiren könnten. Endlich aber existirt das letzte Glied der Reihe, für $\mathbf{X}=\mathrm{CH}_{3}$, das Oxim des Acetophenons, gleich dem Anfangsgliede, dem Oxim der Acetophenoncarbonsäure (= Benzoylessigsäure) nur in einer einzigen, aber in der entgegengesetzten Configuration; das $\mathrm{Oxim} \mathrm{C}_{6} \mathrm{H}_{5}$. $\mathrm{CNOH} . \mathrm{CH}_{3}$ besteht wegen der geringsten Anziehung zwischen Methyl und Hydroxyl nur mit Antistellung dieser beiden Gruppen, das Oxim $\mathrm{C}_{6} \mathrm{H}_{5}, \mathrm{CNOH} . \mathrm{CH}_{2} \mathrm{COOH}$ wegen der stärksten Anziehung zwischen carboxylirten Methyl and Hydroxyl nur mit Synstellung dieser beiden Gruppen.

Derartige Erscheinungen treten nun stets in ähnlicher Art auf, wenn in Molekülen X . CNOH . Y für X ein anderes Radical als constant eingesetzt wird.

Es sei $\mathrm{X}$ das stärkst anziebende Radical $\mathrm{COOH} . \mathrm{CH}_{2}$ : dann werden für alle Oxime $\mathrm{COOH} . \mathrm{CH}_{2}$. $\mathrm{CNOH}$. Y die Configurationen $\mathrm{COOH} . \mathrm{CH}_{2} \cdot \mathrm{C} . \mathrm{Y}$

HO . $\ddot{N}$ stabil, bezw. einzig existenzfähig. Setzt man das in der obigen Skala der Gruppe $\mathrm{COOH} \mathrm{CH}_{2}$ nahestebende $\mathrm{Y}=\mathrm{COOH}$ ein, so resultiren die Oximidobernsteinsäuren $=$ Ketoximessigcarbonsăuren $\mathrm{COOH}$. $\mathrm{CH}_{2}$. CNOH . COOH, bezw. ihre Aethersäuren. Das Carboxyl vermag sich gegenüber der Gruppe $\mathrm{COOH} . \mathrm{CH}_{2}$ noch geitend zu machen, stebt ihm aber an Intensität der Anziehnng auf das Oximhydroxyl nach; denn es ist:

$\mathrm{COOH} \cdot \mathrm{CH}_{2} \cdot \mathrm{C} . \mathrm{COOR}$ HO. $\ddot{\mathrm{N}}$ stabil und

$\mathrm{COOH} . \mathrm{CH}_{2} \cdot \mathrm{C} \cdot \mathrm{COOR}$ $\ddot{N}$. OH labil ${ }^{1}$ ).

Die hinter dem Carboxyl folgenden Gruppen vermögen überhaupt nicht mehr das Hydroxyl auf ibre Seite zu bringen; schon für $X$ $=\mathrm{C}_{6} \mathrm{H}_{5}$, für das Oxim der Benzoylessigsäure, die Phenylketoximessigsäure $\left.{ }^{2}\right) \mathrm{C}_{6} \mathrm{H}_{3} \cdot \mathrm{CNOH} \cdot \mathrm{CH}_{2} \cdot \mathrm{COOH}$ besteht nur die Synstellung der beiden sauerstoffhaltigen Gruppen, und das Gleiche gilt dann natürlich für $\mathrm{X}=\mathrm{CH}_{3}$, für das Oxim der Acetessigsäure, die Methylketoximcarbonsäure $\mathrm{CH}_{3} \cdot \mathrm{CNOH} . \mathrm{CH}_{2} \cdot \mathrm{COOH}^{3}$ ).

Oder man wähle $X=$ Carboxyl, also Oxime COOH.CNOH.Y. Dann ist, wie soeben erwähnt, für $\mathrm{Y}=\mathrm{CH}_{2} \mathrm{COOH}$ die Gegenstellang von Carboxyl und Hydroxyl bevorzugt. Aber schon für $\mathrm{Y}=\mathrm{C}_{6} \mathrm{H}_{5}$ tritt der Umschlag ein; denn von den Phenylketoximcarbonsăuren $\mathrm{COOH} . \mathrm{CNOH} . \mathrm{C}_{6} \mathrm{H}_{5}$ besteht die Configuration

$\mathrm{COOH} . \mathrm{C} \cdot \mathrm{C}_{6} \mathrm{H}_{3}$ $\ddot{\mathrm{N}} . \mathrm{OH}$

1) C. Cramer, diese Berichte XXIV, 1198.

2) A.Hantzsch, diese Berichte XXIV, 502.

3) Ibid. 496 . 
zwar noch, ist aber labil gegenüber

$\left.\mathrm{COOH} . \mathrm{C} \cdot \mathrm{C}_{6} \mathrm{H}_{5}{ }^{1}\right)$
$\mathrm{HO} . \ddot{\mathrm{N}}$ nunmehr für $\mathrm{Y}=\mathrm{C}_{6} \mathrm{H}_{4} \mathrm{X}$, so scheint die Synstellung zwischen Phenyl und Hydroxyl kaum mehr bestehen zu können; wenigstens hat man ${ }^{2}$ ) die Oxime der Piperonyl-, Apiol- and Anisketoncarbonsäure nur in der einzigen Configuration $\begin{gathered}\mathrm{COOH} . \mathrm{C} \cdot \mathrm{C}_{6} \mathrm{H}_{4} \mathrm{X} \\ \mathrm{HO} . \ddot{\mathrm{N}}\end{gathered}$ für $\mathrm{Y}=\mathrm{C}_{4} \mathrm{H}_{3} \mathrm{~S}$, bezw. $\mathrm{CH}_{3}$ war es bereits bekannt, dass die einzig existirenden Formen ebenfalls nur in den Configurationen $\mathrm{COOH} . \stackrel{\mathrm{C}}{\mathrm{C}} \mathrm{C}_{4} \mathrm{H}_{3} \mathrm{~S}\left(\mathrm{CH}_{3}\right)$

$\mathrm{HO} . \ddot{\mathrm{N}}$ bestehen ${ }^{3}$ ).

Setzt man für $X$ die in der obigen Serie nicht aufgenommene Gruppe $\left(\mathrm{C}_{6} \mathrm{H}_{3} . \mathrm{CO} . \mathrm{CH}_{2}\right)$ bezw. $\left(\mathrm{C}_{4} \mathrm{H}_{3} \mathrm{~S} . \mathrm{CO} . \mathrm{CH}_{2}\right)$, so erhält dieselbe nach den Untersuchungen von $\mathrm{Salvatori}^{4}$ ) jedenfalls ihren Platz vor dem Phenyl, und wahrscheinlich sogar vor dem Carboxyl; wenigstens verwandeln sich die Oxime der Acetophenonbezw. Acetothiënonoxalsäure $\mathrm{C}_{6} \mathrm{H}_{5} \cdot\left(\mathrm{C}_{4} \mathrm{H}_{3} \mathrm{~S}\right) \cdot \mathrm{CO} \cdot \mathrm{CH}_{2} \cdot \mathrm{CNOH}$. COOH viel leichter in Oxazolone, als wie in Nitrile, reagiren also viel mehr im Sinne der Configuration $\mathrm{C}_{6} \mathrm{H}_{5}\left(\mathrm{C}_{4} \mathrm{H}_{3} \mathrm{~S}\right)$. CO. $\mathrm{CH}_{2}$. C. COOH als nach $\begin{gathered}\mathrm{C}_{6} \mathrm{H}_{5}\left(\mathrm{C}_{4} \mathrm{H}_{3} \mathrm{~S}\right) \cdot \mathrm{CO} \cdot \mathrm{CH}_{2} \cdot \mathrm{C} \cdot \mathrm{COOH} \\ \ddot{\mathrm{N}} \cdot \mathrm{OH}\end{gathered}$ ziebung dieser Gruppe folgt auch aus ibrer Aehnlichkeit mit der stärkst anziehenden Gruppe $\mathrm{CH}_{2} . \mathrm{COOH}$; denn beide enthalten in Bezug auf dieGruppe CNOHein in $\beta$-Stellung befindliches Carbonyl; $\left(\mathrm{C}_{6} \mathrm{H}_{5} . \mathrm{CO} . \mathrm{CH}_{2}\right.$ vergleichbar $\mathrm{HO}, \mathrm{CO}, \mathrm{CH}_{2}$ ).

Dass Thiophen- (und Furfuran)rest hinter dem Benzolrest reagiren und stereochemisch schon einen Uebergang zu den Alkoholradicalen bilden, ist bekanntlich bereits dadurch nachgewiesen worden, dass die Configuration $\begin{gathered}\mathrm{C}_{4} \mathrm{H}_{3} \mathrm{~S} . \mathrm{C} \cdot \mathrm{Y} \\ \mathrm{HO} . \ddot{\mathrm{N}}\end{gathered}$ besteht ${ }^{5}$ ). Dass die Alkoholradicale im Allgemeinen die geringste Neigung zum Oximhydroxyl besitzen, ist überall bestätigt worden; denn alle Oxime von der Structurformel $\mathrm{C}_{n} \mathrm{H}_{2 \mathrm{n}+1}$. CNOH.Y sind bisher nur in der Gegenstellung zwischen Alkyl und Hydroxyl erhalten worden; z. B. sind die Oxime des Acetophenons, der Lävulinsäure, Brenztraubensäure, Acetessigsäure etc. gleichartig configurirt:

1) A. Hantzsch, diese Berichte XXIV, 41.

2) Ciamician und Garelli, Gazz. chim. XXI, 2, 173.

3) Diese Berichte XXIV, 48.

4) Gazz. chim. XXI, 2, 268 .

5) Diese Berichte XXIV, 47-49, 60. 


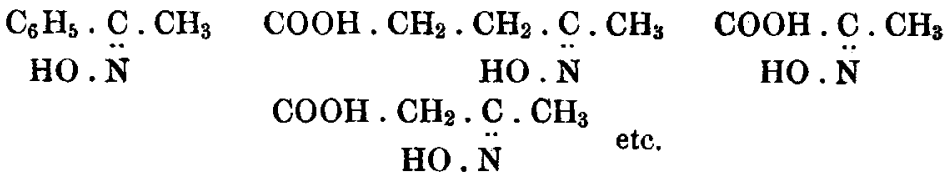

Uebrigens soll um so weniger geläugnet werden, dass die Configurationen $\begin{gathered}\mathrm{CnH}_{2 \mathrm{n}+1} \cdot \mathrm{C} \cdot \mathrm{X} \\ \mathrm{HO} . \ddot{\mathrm{N}}\end{gathered}$

könnten, als die bekanntlich von Franchimont 1 ) zuerst nachgewiesenen, sodann von Dunstan und Dymond ${ }^{2}$ ) untersuchten zwei s Modificationen « des Acetaldoxims vielleicht durch die Configurationen

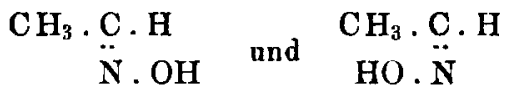

wiedergegeben werden könnten.

Der Einfluss der Alkoholradicale äussert sich ganz ähnlich, nur natürlich schwächer bei grosser Entfernung derselben vom Oximbydroxyl; denn die alkylirten Benzophenonoxime sind ohne Ausnahme labil in der Configuration

$$
\begin{aligned}
& \mathrm{C}_{6} \mathrm{H}_{5}\left.\stackrel{\mathrm{C}}{\mathrm{C}} \cdot \mathrm{C}_{6} \mathrm{H}_{4} \mathrm{C}_{\mathrm{n}} \mathrm{H}_{2 \mathrm{n}+1}{ }^{3}\right) \\
& \mathrm{N} . \mathrm{OH}
\end{aligned}
$$

Dass endlich von allen Alkobolradicalen das Methyl im speciellen die eben besprochene Eigenschaft am ausgeprägtesten zeigt, ist durch die Arbeiten über fette und alkylirte aromatische Ketoxime ansführlich gezeigt worden, so dass hier nur der Vollständigkeit halber daran erinnert werde, dass die fetten Ketoxime von der Structurformel $\mathrm{CH}_{3} . \mathrm{CNOH} . \mathrm{C}_{n} \mathrm{H}_{2 n+1}$ vorwiegend im Sinne der Configuration $\mathrm{CH}_{3} \cdot \mathrm{C} . \mathrm{C}_{\mathbf{n}} \mathrm{H}_{2 \mathrm{n}+1}$ $\ddot{\mathrm{N}}$. OH reagiren ${ }^{4}$ ), dass von allen alkylixten Benzophenonoximen das Phenylsyntolylketoxim $\begin{gathered}\mathrm{C}_{6} \mathrm{H}_{5} \cdot \underset{\mathrm{C}}{\mathrm{C}} \cdot \mathrm{C}_{6} \mathrm{H}_{4} \mathrm{CH}_{3} \\ \stackrel{\mathrm{OH}}{\mathrm{OH}}\end{gathered}$ das labilste darstellt und speciell unbeständiger ist als das analoge Aethylderivat: $\mathrm{C}_{6} \mathrm{H}_{5} \cdot \mathrm{C} \cdot \mathrm{C}_{6} \mathrm{H}_{4} \mathrm{C}_{2} \mathrm{H}_{5}{ }^{5}$ )<smiles>O[V]</smiles>
u. B. w.

Während nach den bisherigen Beispielen die verscbiedenartigsten, an die Gruppe $\mathrm{CNOH}$ gebundenen Radicale stets im Sinne der obigen Reihenfolge die Configuration der Oxime beeinflussen, lässt sich, wie bereits oben erwähnt, der Wasserstoff in diese Serie nicht einfügen. Der Einfluss des Wasserstoffs auf die Configuration ist,
1) Rec. trac. chim. X, 236 .
2) Chem. Zeitung 1892.
3) Diese Berichte XXIV, 4052.
4) Diese Berichte XXIV, 4018.
5) Diese Berichte XXIV, 4052 und 4054. 
wenigstens scheinbar, regellos und gegenüber den anderen, kohlenstoffhaltigen Radicalen in hohem Grade wechselnd; mit anderen Worten, die obigen Regeln gelten zwar für die Ketoxime, dagegen nicht oder nur mit bedeutenden Ausnahmen, für die Configuration der Aldoxime H . CN OH. Y im weitesten Sinne des Wortes. Hier steht der Wasserstoff in vielen Fällen an der Spitze aller übrigen Radicale, und sogar speciell vor der Gruppe $\mathrm{CH}_{2}$. $\mathrm{COOH}$, also im schärfsten Gegensatz zum Methyl. Denn für $\mathrm{X}=\mathrm{CH}_{2} . \mathrm{COOH}$ resultirt die Aldoxinessigsäure $\mathrm{H} . \mathrm{CNOH} \cdot \mathrm{CH}_{2} \cdot \mathrm{COOH}$ (sogen. $\beta$-Oximidopropionsäure, für welche neulich die Configurationsformel HO.

H.C. $\mathrm{CH}_{2}$. $\mathrm{COOH}$

nachgewiesen worden ist "). Die Gruppirung $\begin{gathered}H . \mathrm{C} \\ \text { HO. }\end{gathered}$ ist also ungemein begünstigt, wie denn nicht nur die fetten Aldoxime ${ }^{2}$ ), sondern auch Thiophenaldoxim ${ }^{3}$ ), Benzoylformoxim ${ }^{4}$ ) $\mathrm{C}_{6} \mathrm{H}_{5} . \mathrm{CO} . \mathrm{CNOH} . \mathrm{H}$ u. a., bisher ausschliesslich als Synaldoxime bekannt sind. Allein gegen einige in der Mitte der obigen Scala enthalten Radicale verhält sich der Wasserstoff anders und zeigt eine geringe Anziehungskraft. Für $\mathrm{Y}=\mathrm{COOH}$ ergiebt sich die Aldoximcarbonsäure (Oximidoessigsäure), deren Structurformel H.CNOH.COOH zufolge ibrer Leitfähigkeit ${ }^{5}$ ) aufzulösen ist in die Configurationsformel $\begin{array}{r}\mathrm{H} \\ \stackrel{\mathrm{C}}{\mathrm{N}} \text {. CO OH }\end{array}$ und für $\mathrm{Y}=\mathrm{C}_{6} \mathrm{H}_{5}$ bezw. $\mathrm{C}_{6} \mathrm{H}_{4} \mathrm{X}$, also für die aromatischen Aldoxime ist bekanntlich die Anticonfiguration H. C. $\mathrm{C}_{6} \mathrm{H}_{5}\left(\mathrm{C}_{6} \mathrm{H}_{4} \mathrm{X}\right)$ $\ddot{\mathrm{N}}$. OH stets begünstigt, bezw. einzig stabil 5 und 6 ).

Diese Sonderstellung des Wasserstoffs wird indess um so weniger Wunder nehmen, als dieselbe bekanntlich auch sonst vorhanden ist, und die $W$ asserstoffverbindungen meist ein weniger einfaches Verbalten zeigen, als die entsprechenden kohlenstoff haltigen Verbindungen: Wahrscheinlich spielen bei den Aldoximen als Wasserstoffverbindungen aussen dem elektriscben Gegensatz der Radicale die wohl mit der Kleinheit und Beweglichkeit des Wasserstoffatoms zusammenbängenden intramolekularen Stabilitätsverhältnisse eine grosse Rolle, denen zufolge z. B. die Configuration mit möglichst eentraler Lage des Schwerpunktes der Molekel sehr bevorzugt sein könnte u. s. w.

1) A. Hantzsch, Diese Berichte XXV, 1904.

3) W. Dollfus, Diese Berichte XXV, 1906.

3) A. Hantzsch, Diese Berichte XXIV, 47.

ל) H. G. Söderbaum, Diese Berichte XXIV, 1318.

5) Hantzsch u. Miolati, Zeitschrift physik. Chemie Juliheft. 
Wie die an die Gruppe $\mathrm{CNOH}$ gebundenen Radicale die Configuration im Sinne der bisherigen Entwicklungen beeinflussen, so mässen die Beständigkeitsrerhältnisse der Oxime, bezw. ibrer Derivate natürlich auch darch Veränderungen der Gruppe NOH, d. i. durch Ersatz des Hydroxylwasserstoffs durch andere Radicale verändert werden. Das bedeutet mit anderen Worten die im Grossen und Ganzen immer beobachtete Thatsache, dass die für die Oxime im freien Zustande stabile Configaration ibre Stabilität in Form ihrer Derivate mehr oder minder verändert. Hier ist es sogar im Allgemeinen gestattet, den Gegensata zwischen positiv und negativ beranzuziehen: Oxime, welche in saurer Lösung bezw. in Form negativer Derivate (Säure-Salze, Acetate) stabil sind, werden mehr oder weniger labil in alkalischer Lösung, bez. in Form von Metallsalzen: Also wenn z. B.

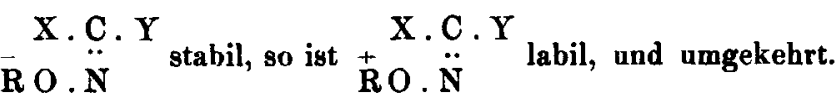

Diese Verbăltnisse erklären auch den von den Gegnern der Stereochemie des Stickstoffs wiederholt gemachten Einwurf, warum die slabilen Oxime überhaupt gebildet werden können. Diese $>$ Labilitätc gilt eben hänfig nur für die freien Oxime, nicht aber für diejenigen ihrer Derivate, als welche sie erzeugt werden. Lässt man z. B. die Einwirkang von Hydroxylamin auf Ketone in stark alkalischer Lösung vor sich gehen, so entstehen die slabilen * Oxime gewisser substituirter Benzopbenone, bezw. des Benzils in Gestalt ihrer stabilen Natronsalze, und zwar um so reichlicher, je stärker alkalisch die Reactionsfüssigkeit ist; so lagern sich die im freien Zustande stabilen Oxime der Phenylglyoxylsäure bezw. des Oxybenzophenons durch Kochen mit Alkali in die im freien $\mathrm{Zu}$ stande labilen, aber als Natriumverbindungen stabilen Stereoisomeren um; so tritt natürlich die umgekehrte Tendenz durch Säuren zu Tage: dieselben wirken, und $\mathbf{z w a r}$ jedesfalls proportional ihren Affinitätsconstanten, je nach der Natur des Oxims mehr oder minder umlagernd auf die alkalistabile Configuration. So hat man z. B. für die oben genannten Oxime die Beziehungen:

$$
\begin{gathered}
\left.\mathrm{C}_{6} \mathrm{H}_{5} \cdot \underset{\mathrm{N}}{\mathrm{C}} \cdot \mathrm{COOH} \underset{\mathrm{OH}}{\mathrm{HCl}} \underset{\mathrm{NaOH}}{\mathrm{NaOH}} \mathrm{C}_{6} \mathrm{H}_{5} \cdot \stackrel{\mathrm{C}}{\mathrm{Cl}} \cdot \mathrm{COONa}^{1}\right) \\
\text { Oxime der Phenylglyoxylsăure }
\end{gathered}
$$

1) Die Umwandlung der Phenylsyn- in Phenylanti-Ketoximcarbonsăure durch kochendes Alkali ist von Russanow beobachtet worden. 


$$
\begin{gathered}
\mathrm{HO} \cdot \mathrm{C}_{6} \mathrm{H}_{4} \cdot \underset{\mathrm{C}}{\mathrm{C}} \cdot \mathrm{C}_{6} \mathrm{H}_{5} \underset{\mathrm{NaOH}}{\mathrm{N}} \stackrel{\mathrm{OH}}{\mathrm{NaO}} \cdot \mathrm{C}_{6} \mathrm{H}_{4} \cdot \underset{\mathrm{HCl}}{\mathrm{C}} \cdot \mathrm{C}_{6} \mathrm{H}_{3} \\
\text { Oxime des } p \text {-Oxybenzophenons }
\end{gathered}
$$

wobei die Leichtigkeit des gegenseitigen Ueberganges gerade dieser Oxime jedenfalls durch ihren Sauerstoffreichthum begünstigt wird. ${ }^{1}$ )

So lassen sich die alkali-stabilen Configurationen bei den freieu Oximen am leichtesten unverändert erhalten, wenn man die alkalische Lösung nicht durch Mineralsäuren, sondern durch Kohlensäure fällt.

Derartige Beziehungen treten besonders deutlich auch bei den Glyoximen X.CNOH.CNOH.Y hervor. Dieselben bestehen bisher häufig nur in zwei Configurationen, indem dann die Configuration der Gruppe X . CNOH veränderlich, die der Gruppe CNOH . Y nnveränderlich ist; sie lassen sich daher gewissermaassen als stereoisomere Monoxime von der Form (X). CNOH.(CNOH.Y) betrachten im Sinne der Raumformeln

$$
\begin{array}{r}
\text { X.C.CNOH.Y } \\
\text { HO. }
\end{array} \text { und } \quad \underset{\text { N.CN }}{\text { N.OH }}
$$

und hier ist bisher ausnabmslos die erstere Configuration die im freien Zustande, bezw. gegen Säuren stabile, und die letztere die im freien Zustande labile bezw. gegen Alkali stabile. Lässt man daher Hydroxylamin in alkalischer Lösung einwirken auf Benzilmonoxim $\mathrm{C}_{6} \mathrm{H}_{5}$. CO . CNOH . $\mathrm{C}_{6} \mathrm{H}_{5}{ }^{2}$ ), auf Phenylglyoxal, $\mathrm{C}_{6} \mathrm{H}_{5} . \mathrm{CO} . \mathrm{CO} . \mathrm{H}^{3}$ ) anf Chloral $\mathrm{CCl}_{3}, \mathrm{CHO}{ }^{4}$ ), auf Dibrombrenztraubensäure $\mathrm{CHBr}_{2}$. $\mathrm{CO} . \mathrm{COOH}^{5}$ ), auf Diketobernsteinsäure $\mathrm{COOH} . \mathrm{CO} . \mathrm{CO} . \mathrm{COOH}^{6}$ ) anf Oximidoacetessigäther $\mathrm{CH}_{3}, \mathrm{CO} . \mathrm{CNOH}$. COOR ${ }^{7}$ ), so erhält man beim vorsichtigen Ansäuern (allfällig durch Ausfällen mit Kohlensäure) überall zuerst die analog configurirten flyoxime

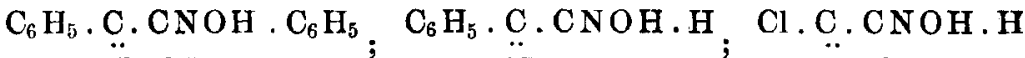

$$
\begin{aligned}
& \text { N. OH } \\
& \text { N. OH } \\
& \text { N. OH }
\end{aligned}
$$

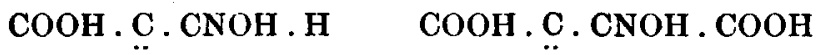

$$
\begin{aligned}
& \text { N. OH } \\
& \text { N . } \mathrm{OH}
\end{aligned}
$$

$\mathrm{COOH} \cdot \mathrm{C} \cdot \mathrm{CNOH} \cdot \mathrm{CH}_{3}$

$$
\ddot{\mathrm{N}} \text {. OH etc. }
$$

1) Ueber den Einfluss der Radicale anf die Leichtigkeit der Configurationsănderung, s. diese Berichte XXIV, 4056.

2) v. Meyer, diese Berichte XXII, 547.

3) Russanow, diese Berichte XXIV, 3497.

4) A. Hantzsch, diese Berichte XXV, 701 .

5) Söderbanm, diese Berichte XXV, 904.

6) Söderbaum, diese Berìchte XXIV, 1215 .

7) Nussberger, vorhergebende Abhandlung. 
welche in freiem Zustande mehr oder minder leicht, am raschesten durch Salzsäuregas in die entgegengesetzt configurirten Stereoisomeren

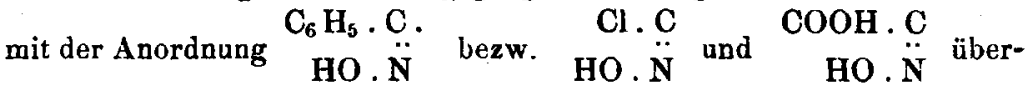
geben.

II. Einfluss der Configuration (und dadurch indirect der Constitution) auf die intramolecularen Reactionen asym metrischer Oxime.

Sehr bemerkenswerth und bisher noch nicht genügend bervorgehoben ist der Umstand, dass nicht nur die Configuration selbst, sondern auch das chemische Verhalten stereoisomerer Oxime von analoger Configuration dureh die Natur der Radicale regelmässig in demselben Sinne beeinflusst wird. Zur Erklärung bezw. Verdeutlichung dieses Umstandes kann man ron folgender Betrachtung ausgehen:

Die obige Reibenfolge der Radicale in Bezug auf ihre grössere oder geringere Anziehung des Hydroxyls tritt nicht nur in der verschiedeneu Stabilität der beiden Configurationen

$$
\begin{array}{rlr}
\text { X.C. } & \text { X } & \text { X.C.Y } \\
\text { HO. } . ~ & \text { and } & \ddot{N} \cdot O H
\end{array}
$$

zu Tage, wonach das Hydroxyl einmal dem $Y$, das andere Mal dem $\mathbf{Y}$ näher erscheint, aber in derselben absoluten Entfernung zu beiden zu stehen scheint; sondern wechselt auch die absolute Entfernung des Hydroxyls von den beiden Radicalen je nachder Stärke ihrer Anzjehung. Bedeute z. B. $X$ eine stark und $Y$ eine schwach anziehende Gruppe, so könnten die beiden Stereoisomeren durch folgende Configurationen versinnbildlicht werden:<smiles>[Y]C([Y])=NO</smiles>

Berücksichtigt man nun die obige Wirksamkeits-Skala, so sind danach beispielsweise Oxime von der Formel COOH. $\mathrm{CH}_{2}$. CNOH. Y und $\mathrm{C}_{\mathrm{n}} \mathrm{H}_{2 \mathrm{n}+1}$. CNOH. $\mathrm{Y}$ im Allgemeinen durch die Configurationen<smiles>[Y]C1=NOC(C(=O)O)C1</smiles>
und<smiles></smiles>

darzustellen, während bei Oximen von der Formel $\mathrm{C}_{6} \mathrm{H}_{5}$. CNOH . Y im allgemeinen mittlere Lagen des Oximbydroxyls vorhanden sein werden, die natürlich ebenso wie die obigen wiederum von der Natur des Radicales $Y$ beeinflusst werden. 
Das chemische Verhalten derartiger Oxime führt in der That zu einer solchen Auffassung, und zwar wieder zu Folge des Princips der intramolecularen Reactionen und speciell der intramolecularen Zersetzung. Wie gemäss demselben im Allgemeinen durch die gegenseitige Reaction zweier Gruppen (Nitrilbildung von Aldoximen, Anhydridbildung von Ketoximsäuren bezw. Glyoximen, Umlagerung stereoisomerer Ketoxime in structurisomere Säureanilide etc.) auf die räumliche Nähe dieser Gruppe zu schliessen ist, so kann man ans der grösseren oder geringeren Leichtigkeit, mit welcher sich derartige Reactionen innerbalb des Moleküles vollziehen, auf die grössere oder geringere Näbe der betheiligten Gruppen schliessen.

Diese wird nun wieder von den im Molekül vorhandenen, bei dieser Zersetzung selbst unbetheiligten Gruppen in dem oben angedenteten Sinne beeinflusst. Dies werde zuerst gezeigt bei dem

1) Zerfall der Synaldoxime in Nitril und Wasser:

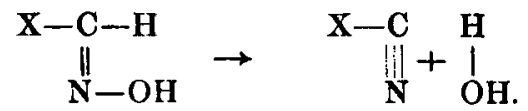

Diese Reaction erfolgt mit sehr verschiedener Leichtigkeit, bald schon bei den freien Oximen, bald nur bei ihren Acetaten, und bei diesen wiederum bald freiwillig, bald erst durch Sodalösung u. s. w.

Diese Vorgănge sind nun abhängig von der Natur des Radicales $\mathrm{X}$; je weniger dasselbe das Oximhydroxyl anziebt, je mehr es also am Ende der obigen Skala steht, um so mebr wird der Wasserstoff das Hydroxyl in seine Nähe bringen, am so leichter werden diese beiden Gruppen als Wasser austreten.

Man vergleiche also die Synaldoxime $\begin{aligned} & \text { X.C.H } \\ & \ddot{N} . O H\end{aligned}$ auf ihre Nitrilbildung, indem man, obiger Skala von rechts nach links folgend, setzt $\mathrm{X}: \mathrm{CH}_{3}, \mathrm{C}_{6} \mathrm{H}_{5} \mathrm{CH}: \mathrm{CH}$ (in obiger Reihe nicht aufgenommen), $\mathrm{C}_{4} \mathrm{H}_{3} \mathrm{~S}$, $\mathrm{C}_{6} \mathrm{H}_{5}, \mathrm{COOH}$. $\mathrm{CH}_{2}$.

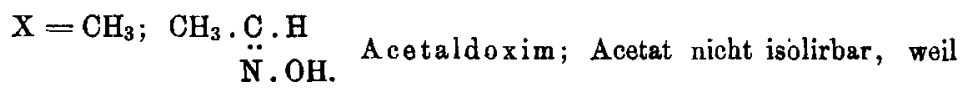

spontan in Acetonitril übergehend. Essiganhydrid spaltet also das Oxim bereits bei gewöhnlicher Temperatur.

$$
\mathrm{X}=\mathrm{C}_{8} \mathrm{H}_{7} ; \mathrm{C}_{6} \mathrm{H}_{5} . \mathrm{CH}: \mathrm{CH} . \underset{\mathrm{N}}{\mathrm{C}} . \mathrm{H} \text {. } \mathrm{H} \text {. Zimmtsynaldoxim. Acetat iso- }
$$

lirbar, jedoch langsam bei gewöhnlicher Temperatur Nitril bildend. Die Existenz des Acetats wird wohl durch die Doppelbindung des Zimmtradicales ermöglicht, welche es dem Benzolrest bereits etwas ähnlicher macht.

$$
\mathrm{X}=\mathrm{C}_{4} \mathrm{H}_{3} \mathrm{~S} ; \mathrm{C}_{4} \mathrm{H}_{3} \mathrm{~S} . \mathrm{C} . \mathrm{H}
$$

$$
\ddot{\mathrm{N}} . \mathrm{OH} \text {. }
$$

Thiophensynaldoxim. Acetat iso-

lirbar, aber nicht sehr haltbar. Das freie Oxim liefert bereits beim Kochen mit Sodalösung Thiophennitril. 


$$
\mathrm{X}=\mathrm{C}_{6} \mathrm{H}_{5} ; \mathrm{C}_{6} \mathrm{~B}_{5} . \underset{\text { N.OH. }}{\mathrm{C} . \mathrm{H}} \text { Benzsynaldoxim. Acetat, wenn isolirt, }
$$
auch ziemlich haltbar; z. B. aus indifferenten Lösungsmitteln umzukrystallisiren; wird aber sohon mit kalter Sodalösang in Benzonitril übergeführt.

$\mathrm{X}=\mathrm{COOH} . \mathrm{CH}_{2} ; \mathrm{COOH} . \mathrm{CH}_{2} \mathrm{C} . \mathrm{H}$

N . OH. Synaldoximessigsäare. Acetat bestündig, und weder durch Soda noch durch Natron in Cyanessigsāure zu. spalten, sondern einfach zur Aldoximsäure sich verseifend.

Aus dieser graduell sich steigernden Beständigkeit der ConC. $\mathrm{H}$

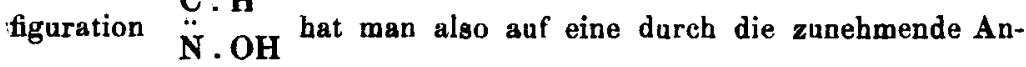
riehung der Radicale $\mathbf{X}$ anf den Wasserrest hervorgerufene graduell zunebmende räumliche Entfernung des Hydroxyls vom Aldehydwasserstoff zu schliessen, im Sinne der Configurationsformeln:<smiles></smiles>

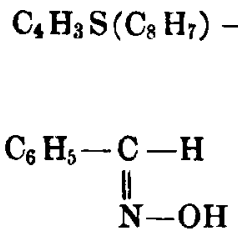<smiles>O=C(O)C/C=N/O</smiles>

2) Der Zerfall der $\alpha$-Ketoxymsyncarbonsäuren in $\mathrm{Ni}$ trile, Kohlensäure und Wasser.<smiles>[X]C(=NO)C(=O)O</smiles>

welcher ebenfalls besonders in Form der Acetate erfolgt, also durch Essiganhydrid eingeleitet wird, ändert sich ebenso regelmässig je nach Natur des Radicales $X$, bezw. seiner Stellung in obiger Skala. Man setze der Reibe nach für $\mathrm{X}=\mathrm{CH}_{3}, \mathrm{C}_{4} \mathrm{H}_{3} \mathrm{~S}, \mathrm{C}_{6} \mathrm{H}_{5}, \mathrm{CH}_{2}, \mathrm{COOH}$ und $\mathrm{H}$.

- Für $\mathrm{X}=\mathrm{CH}_{3}$ resultirt das Oxim der Benztraubensāure, die Methylsynketoximcarbonsäure. Das Acetat ist durch Essiganhydrid nicht zu erbalten; es zerfällt spontan in Essigsäure, Koblensäure und Acetonitril.

Für $\mathrm{X}=\mathrm{C}_{4} \mathrm{H}_{3} \mathrm{~S}$ erhält man das Oxim der Thiënylglyoxylsäure, deren Acetat auf dieselbe Weise isolirt werden kann, aber höchst unbeständig ist.

Für $\mathrm{X}=\mathrm{C}_{6} \mathrm{H}_{5}$ ergiebt sich das Oxim der Phenglglyoxyl: \&ãure (Phenylsynketoximcarbonsäure). Dasselbe zerfällt beim Behandeln mit Essiganhydrid nur partiell in Benzonitril; das einmal gebildete Acetat lässt sich vorsichtig amkrystallisiren und zerfällt erst in Soda in analoger Weise. 
Für $\mathrm{X}=\mathrm{COOH} . \mathrm{CH}_{2}$ resultirt die Ketoximessigsyncarbonsäare (Ebert's $\alpha$-Oximidobernsteinsäure),

$$
\begin{array}{r}
\mathrm{COOH} \cdot \mathrm{CH}_{2} \cdot \underset{\mathrm{N}}{\mathrm{C}} \cdot \mathrm{COOH}, \\
\mathrm{OH},
\end{array}
$$

welche durch Essiganhydrid nicht mehr in analoger Weise zersetzt, d. i. nicht in Cyanessigsäure verwandelt wird.

Für $X=H$ resultirt Oximid oessigsä ure $=$ Aldoximcarbonsāure, welche durch Essiganhydrid ein so beständiges Acetat bildet, dass dasselbe nicht einmal mebr durch Soda in analoger Weise - d. i. in Cyanwasserstoff, Kohlensäure und Wasser - zerfällt.

Wieder wird also durch die das Oximbydroxyl am wenigsten arziehenden zu ihm in Gegenstellung befiadlichen Radicale die intramoleculare Zersetzung befördert, durch die stärker anziehenden in gleicher Stellung diese Zersetzung verringert; im Sinne der Configurationsformeln :

$$
\mathrm{CH}_{3}\left(\mathrm{C}_{4} \mathrm{H}_{3} \mathrm{~S}\right)-\mathrm{C}-\mathrm{COOH}
$$<smiles>CCCCCC(CCOC(C)=O)=NO</smiles>

Der genaueste Einblick in derartige intramoleculare Entfernung $\$-$ verbältnisse ist durch den Vergleich der von der Atomanordnung am meisten beeinflussten Eigenschaft, der Leitfähigkeit, bezw. der Affinitätsconstanten erbalten worden; vorläufig allerdings nur betr. der Wirkung. der verschiedenen Alkoholradicale im Molekül der $\alpha$-Ketoximsäuren von der Structurformel $\mathrm{C}_{n} \mathrm{H}_{2 n+1}$. $\mathrm{CNOH}$. COOH. Die Affinitätsconstanten nehmen in dieser homologen Reihe nicht regelmässig, sondern sprungweise $a^{1}$ ) was wieder auf eine sprungweise wechsel nue Wirkung des Alkyls zurückzuführen ist und verdeutlicht werden kann durch die Formeln:<smiles>O=C(O)/C=N\O</smiles><smiles>CC(=NO)C(=O)O</smiles><smiles>CCC(=NO)C(=O)O</smiles><smiles>CCCC(=NO)C(=O)O</smiles>

1) Diese Berichte XXIV, 498 und 504. 
3) Die Anhydrisirung der $\beta$-Ketoximsyncarbonsăuren zu Synoxazolonen:

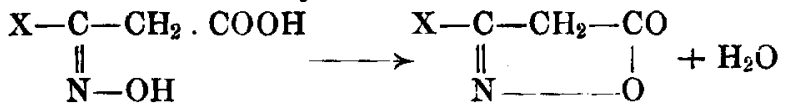

Auch diese intramoleculare Ausscheidung von Wasser vollzieht sich um so leichter, je weniger kräftig das Radical $X$ auf das Oximhydroxyl wirkt, je unumschränkter also das Radical $\mathrm{CH}_{2}$. $\mathrm{COOH}$ wirken kann. Da diese letztere Gruppe in der Wirksamkeitsskala an der Spitze steht, so vermag nicht nur durch $\mathrm{X}=\mathrm{CH}_{3}$, sondern selbst durch $\mathrm{X}=\mathrm{C}_{6} \mathrm{H}_{5}$, d. i. nicht nur für Methyl-, sondern selbst für Phenylsynketoximessigsäure die spontane Anhydrisirung nicht verbindert zu werden; es entstehen augenblicklich Metbyl- bezw. Phenylsynoxazolon, wobei sich indess doch der etwas kräftigere Einfluss des Phenyls durch die etwas geringere Beständigkeit dieses letzteren Ringderivates äussert; denn Phenylsynoxazolon wird z. B. durch Säuren ${ }^{1}$ ) und auch durch Kochen mit Baryt langsam in Kohlensäure und Acetophenonoxim gespalten, während das Methylsynoxazolon unter gleichen Bedingungen noch nicht angegriffen, $d$. i. aufgespalten wird.

Setzt man indess für $\mathrm{X}=\mathrm{COOH}$, also das nächst der Gruppe $\mathrm{CH}_{2}, \mathrm{COOH}$ stärkst wirkende Radical, so anhydrisirt sich die so resultirende Ketoximsynessigcarbonsäure (sogen. $\beta$-Oximidobernsteinsäure, nicht zu Oxazoloncarbonsäure, und ebenso wenig ibre Aethersäure; das Carboxyl verhindert also in Gegenstellung zum Oximhydroxyl, dass das letztere in die zur Anhydrisirung erforderliche Nähe der Gruppe. $\mathrm{CH}_{2} \mathrm{COOH}$ gelange; man darf dies also wieder durch die Raumbilder darstellen:<smiles>CCCCC(CC(=O)O)=NC(=O)OC(CC(=O)O)C(=O)O</smiles>

Setzt man für $X$ das in obiger Reihe nicht enthaltene Acetyl, so erhält man die jüngst von $\left.\mathrm{Thal}^{2}\right)$ beschriebene $\beta$-Oximidolävulinsäure $k=$ Acetylketoximessigsäure $\mathrm{CH}_{3} \cdot \mathrm{CO} \cdot \mathrm{CNOH} \cdot \mathrm{CH}_{2} . \mathrm{COOH}$, welche wahrscheinlich ebenfalls die Configuration $\mathrm{CH}_{3}, \mathrm{CO} . \mathrm{C} \cdot \mathrm{CH}_{2} \cdot \mathrm{COOH}$ N. $\mathrm{OH}$

besitzen dürfte, sich aber auch nicht anhydrisirt. Das sauerstoffreiche Acetyl ist eben dem Carboxyl vergleichbar, $\left(\mathrm{CO} . \mathrm{CH}_{3}\right.$ ähnlich $\mathrm{CO} . \mathrm{OH})$ und wird daher seine Stelle jedenfalls in der obigen Reihe nabe hinter dem Carboxyl und ziemlich weit vor den Alkobolradicalen erhalten. Dem entsprechend verhindert es noch, wie das Carboxyl, die Anhydrisirung; verwandelt man es aber in $\mathrm{CH}_{3} . \mathrm{CNOH}$, so ist,

y) Zeitschr. f. phys. Chem. 1892, Juliheft.

2) Diese Berichte XXV, 1719. 
wie derselbe Antor angiebt, die Anhydrisirung wieder möglich; denn die Säure $\mathrm{CH}_{3} . \mathrm{CNOH} . \mathrm{CNOH} . \mathrm{CH}_{2} . \mathrm{COOH}$ besteht in dieser Form.

4) Dem Verhalten der $\beta$-Ketoximsäuren ganz ähnlich ist die besonders von Claisen ${ }^{1}$ ) studirte Anhydrisirung der $\beta$-Oximidoket one von der Formel X. CNOH. $\mathrm{CH}_{2}$. CO.R zu Synoxazolen

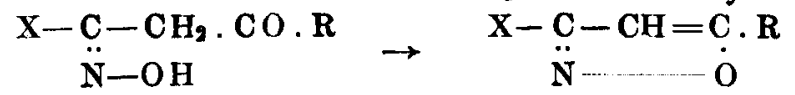

Die sich für $\mathrm{X}=\mathrm{CH}_{3}$ bezw. $\mathrm{C}_{6} \mathrm{H}_{5}$ ergebenden Monoximedes Acetylacetons $\mathrm{CH}_{3} . \mathrm{CNOH} \cdot \mathrm{CH}_{2} \cdot \mathrm{CO} \cdot \mathrm{CH}_{3}$ bezw. Dibenzoylmethans $\mathrm{C}_{6} \mathrm{H}_{5} \cdot \mathrm{CNOH} . \mathrm{CH}_{2} . \mathrm{CO} \cdot \mathrm{C}_{6} \mathrm{H}_{5}$ sind ebensowenig im freien Zustande zu erbalten, als wie Oxime der Acet- bezw. Benzoylessigsäure; man erhält spontan Dimethyl (bezw. Diphenyl)-Synoxazol.

Setzt man aber für $\mathrm{X}=\mathrm{COOH}$, so sind die zu dieser Gruppe gehörigen Oxime der Acetophenon- bezw. Acetothiënonoxalsäure $\mathrm{COOH} . \mathrm{CNOH} . \mathrm{CH}_{2}$. CO. $\mathrm{C}_{6} \mathrm{H}_{5}$ bezw. $\mathrm{COOH} . \mathrm{CNOH} . \mathrm{CH}_{2} . \mathrm{CO} . \mathrm{C}_{4} \mathrm{H}_{3} \mathrm{~S}$ im freien Zustande bekannt, and obgleich ihre Configuration noch nicht bestimmt worden ist, so bedeutet doch ihre Existenz, dass das Anhydrisirungsbestreben $z u$. Oxazolcarbonsäure durch den starken Einfluss des Carboxyls zwar nicht aufgeboben, aber doch rerringert worden ist ${ }^{2}$ ).

Setzt man endlich für $X=H$, so erhält man Ketoaldoxime, z. B. das Oxim des Acetessig- und Benzoylessigaldehyds $\mathrm{H}$. $\mathrm{CNOH} . \mathrm{CH}_{2}$. $. \mathrm{CO} . \mathrm{CH}_{3}$ and $\mathrm{H} . \mathrm{CNOH} . \mathrm{CH}_{2} \cdot \mathrm{CO} . \mathrm{C}_{6} \mathrm{H}_{3}$. Hier bestehen, entm sprechend der grossen Begünstigung der Configuration $\begin{array}{r}\text { H.C } \\ \text { HO.N }\end{array}$ diese Aldoxime nicht nur in freiem Zustande, sondern sogar, wie die ibnen vergleichbare Aldoximessigsäure $\mathrm{H} . \mathrm{CNOH} \cdot \mathrm{CH}_{2}$. $\mathrm{CO} . \mathrm{OH}$, in der den Alkylderivaten entgegengesetzten Configuration H. C. $\mathrm{CH}_{2} \cdot \mathrm{CO} . \mathrm{CH}_{3}\left(\mathrm{C}_{6} \mathrm{H}_{5}\right)$; HO. $\ddot{\mathrm{N}}$

Sinne dieser Raumformel, indem sie leicht in Cyanide übergeben: C. $\mathrm{CH}_{2}, \mathrm{CO} . \mathrm{CH}_{3}\left(\mathrm{C}_{6} \mathrm{H}_{5}\right)$, $\dddot{N}$

und nur indirect im Sinne der Configuration

$\mathrm{H} . \mathrm{C} . \mathrm{CH}_{2} \cdot \mathrm{CO} \cdot \mathrm{R}$, $\ddot{N}$. OH

wirkenden Acetylchlorids sich zu den Synoxazolen anhydrisiren ${ }^{3}$ ).

indem sie nur unter Vermittelung des umlagernd H. $\mathbf{C}-\mathrm{CH}=\mathrm{CR}$ $\ddot{\mathbf{N}}-\dot{\mathbf{O}}$

1) Diese Berichte XXIV, 3901.

2) Vergl. daselbst 3908 und besonders Gazz. Chim. XXI, 2, 268.

3) Claisen, diese Berichte XXV, 130 und Hantzsch, daselbst 505. 
5) Ueber die Anbydrisirung von $\gamma$-Ketoximsä u ren ist $z$. Th. schon von W. Dollfuss berichtet worden; nm die Abbängigkeit dieser Reaction von Constitution und Configuration weiter auszuführen, so werde zunächst daran erinnert, dass die echten gesättigten $\gamma$-Ketoximsäuren von der Formel X . CN O H $. \mathrm{CH}_{2}, \mathrm{CH}_{2} . \mathrm{COOH}$, d. i. die Oxime der Aceto- und Benzoylpropionsäure sich bisher nicht baben anhydrisiren lassen, obgleich sie in Sinne der Configuration $\mathrm{CH}_{3}\left(\mathrm{C}_{6} \mathrm{H}_{5}\right) \cdot \mathrm{C} . \mathrm{CH}_{2} \cdot \mathrm{CH}_{2} \cdot \mathrm{COOH}$

$$
\ddot{\mathrm{N}} \text {. } \mathrm{OH}
$$

und Oximhydroxyl besitzen; der Grund hierfür liegt erstens darin, dass diese beiden Gruppen bereits an sich relativ weit von einander entfernt sind, zweitens aber noch mehr wohl darin, dass diese durch drei Kohlenstoffatome getrennten Gruppen in Folge der freien Drehbarkeit dieses Systems jedenfalls in Wirklichkeit noch viel entfernter von einander liegen; was durch die obige Formel nicht direct, am räumlichen Tetraëdermodell indess sofort zu erkennen ist. Dieser Vorstellung entsprechend steigt die Anhydrisirungstendenz der $\gamma-\mathrm{Ke}-$ toximsäuren, wenn durch Doppelbindungen zwischen zwei Kohlenstoffatomen die Gruppen $\mathrm{COOH}$ und $\mathrm{NOH}$ in plansymmetrische, maleinnoide Stellung gerathen und dadurch am »Ausweichen« rerhindert werden; $\gamma$-Ketoximsäuren von der Form<smiles>[Y]C(=O)/C=C\C(=O)O</smiles>
werden also der Anbydridbildung fähig sein. Und in der That finden wir dieselbe bei den der Benzolreihe zugehörigen $\gamma$-Ketoximsäuren von der allgemeinen Form

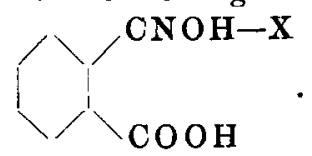

Allein hier kommt

nunmebr wieder als zweiter Factor die Natur des Radicales $\mathrm{X}$ in Betracht. Die fur $\mathrm{X}=\mathrm{CH}_{3}$ und $\mathrm{C}_{6} \mathrm{H}_{5}$ resultirenden Oxime der Acetophenon- und Benzophenonorthocarbonsäure $\mathrm{C}_{6} \mathrm{H}_{4}<\mathrm{CNOH} . \mathrm{CH}_{3}\left(\mathrm{C}_{6} \mathrm{H}_{5}\right)(1)$ bestehen wie die $\beta$-Oximidosäuren überhaupt nur als innere Anhydride; setzt man indess für $\mathrm{X}=\mathrm{H}$, so besteht die so resultirende Benzaldoximorthocarbonsäure (das Oxim der Phtalaldehydsăure) ${ }^{1}$ ) in freiem Zustande: $\mathrm{C}_{6} \mathrm{H}_{4}<\mathrm{COOH}$, weil der Wasserstoff, wieder im Gegensatz zum Methyl, das Oximhydroxyl in grössere Nähe zu sich selbst, also in grössere Entfernung zum Carboxyl bringt und deshalb die Anbydridbildung verhindert. Aehnliches könnte auch für die

1) Racine, Ann. Chem. Pharm. 239, 85. 
complicirteren Oxime von Benzi]- und Desoxybenzoïncarbonsäuren ${ }^{1)}$ ausgefübrt werden.

6) Die Anhydrisirung von Synglyoximenzu Synazoxazole n (Furazanen)

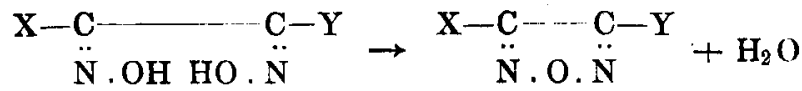

ist ebenfalls von der Natur der Radicale $X$ und $Y$ abhängig, und tritt bekanntlich nur unter bestimmten Verbältnissen ein. Da dieselben indess noch nicht eingehender bekannt sind und gerade nach dem bisher Bekannten besonders complicirt liegen (weil hier nicht nur ein, sondern zwei Radicale die Configuration der Oximidogruppen und damit auch die intramoleculare Reaction beeinflussen), soll hierauf an dieser Stelle noch nicht eingegangen werden ${ }^{2}$ ).

7) Die graduell verschiedene Wirkungsweise der Radicale auf die absolute räumliche Entfernung der Atome im Molekül von Oximen und damit auf ihre Ueberführung in stickstoff haltige Ringe äussert sich natürlich auch im amgekehrten Sinne bei den A ufspaltungen dieser Ringe; sie beeinflusst deren Beständigkeit in hohem Grade. So ist, wie bereits oben bremerkt, der Ring der Synoxazolone $\mathrm{X} \cdot \underset{\mathrm{N}}{\mathrm{C}} \cdot \mathrm{CH}_{2} \cdot \underset{\mathrm{O}}{\mathrm{C}}$ bisher überhaupt kaum zu öffuen, wenn $\mathrm{X}=\mathrm{CH}_{3}$; schwer aufzuspalten, wemn $\mathrm{X}=\mathrm{C}_{6} \mathrm{H}_{5}$; und spontan aufzuspalten (d. i. bisher überhaupt nicht zu erhalten), wenn $\mathrm{X}=\mathrm{COOH}$ and $\mathrm{X}=\mathrm{H}$.

Der Ring der Synoxazole $\mathrm{X} \cdot \underset{\mathrm{N}}{\mathrm{C}} \cdot \mathrm{CH}: \underset{\mathrm{O}}{\mathrm{C}}$ ist an sich $\mathrm{zwar}$ beständiger, aber doch auch von veränderlicher Stabilität; so ist er für $\mathrm{X}=\mathrm{CH}_{3}$ oder $\mathrm{C}_{6} \mathrm{H}_{5}$ sehr stabil, für $\mathrm{X}=\mathrm{COOH}$ weniger stabil und für $\mathrm{X}=\mathrm{H}$ sehr labil, ähnlich, aber wieder complicirter, liegen die Verbältnisse für den Ring der Azoxazole (Furazane).

Die hier hervorgehobenen Erscheinungen lassen sich, ausschliesslich auf die Alkoholradicale bezogen, auch so ausdrücken, dass die Alkyle als Beförderer der Ringschliessung, oder als Schützer der Ringe erscheinen. Diese Wirkungsweise ist übrigens durchans nicht auf das Gebiet der Oxime beschränkt. Sie gilt bekanntlich in ähnlicher Weise auch für stickstoff freie und nicht in Stereoisomeren rorkommendeVerbindungen, wie dies vor Allem durch die bereits eingangs erwähnten Untersuchungenivon V.M eyer, A uwers, C. Bis c ${ }^{\prime}$ off u. a. ${ }^{3}$ ) für die Alkylderivate von Dicarbonsäuren und $\gamma$-Ketonsäuren nachgewiesen

1) Graebe, diese Berichte XXIII, 1344.

2) Vergl. diese Berichte XXV, 705 und die vorangehende Arbeit von Nussberger.

3) Diese Berichte XXIV, 101, 620, 1599, 2414 n. a. 0. 
worden ist. Bei diesen wird die Tendenz zum Zerfall in Wasser und Anhydrid, bezw. Lacton, bei den Oximen diejenige zum Zerfall in Wasser und Nitril, bezw. Oxazol durch Alkyle befördert. Die Alkoholradicale begünstigen also indirect die intramolecularen Zersetzungen, indem sie diejenigen Configurationen herzustellen streben, bezw. diejenigen Gruppen einander nähern, durch deren gegenseitige Reaction aus einem Moleküle deren zwei gebildet werden.

Zürich,.in Juni 1882.

\section{J. Alts chul: Nachtrag zu der Abhandlung über $p$-Oxalkylderivate des Phenylhydrazins etc. \\ (Eingegangen am 27. Juni.)}

Zu obiger Arbeit ${ }^{1}$ theilt mir Hr. Dr. Groll mit, dass er die von mir als Vorproducte beschriebenen Salze $p$-äthoxydiazobenzolsulfonsaures- und $p$-äthoxyphenylhydrazinsulfonsaures Natrium bereits früher erhalten und in seiner Dissertation (Basel 1888) beschrieben hat. Hr. Dr. Groll bat auch versucht, durch Einwirkung wăssriger Salzsăure auf letzteres Salz zum Aethoxyphenylhydrazin zu gelangen, hat jedoch gleich mir hierbei negative Resultate erhalten und seine Versuche damals abgebrochen.

Betreffs der obigen Salze findet sich in der betreffenden Dissertation die unrichtige Angabe, dass sich dieselben nicht unzersetzt wasserfrei erhalten liessen. Dr. G roll schliesst ans seinen Analysen, dass beide Salze, die er nur im Exsiccator trocknete, ein Molekül Krrystallwasser enhalten. Doch stimmt thatsāchlich seine Natriumbestimmung des $p$-ăthoxydiazobenzolsulfonsauren Natriums sehr viel besser auf ein wasserfreies Salz.

Es berechnet sich näulich

für das wasserfreie Salz . . . . . . $9.1 \mathrm{pCt}$. Natrium

》 Salz mit 1 Molekül Wasser . . 8.5 》 》

während Hr. Dr. Groll findet . . . 8.9 » ”

Ueberdies habe ich gezeigt, dass beide Salze sich unzersetzt trocknen lassen und habe die betreffenden Analysen mitgetheilt.

Ich benutze die Golegenheit, eine in meiner Abhandlung durch ein Versehen fortgelassene Literaturangabe hier nachzutragen. Auf Seite 1852 fehlt die auf die Analyse ron Salipyrin bezügliche Fussnote: "Pharm. Centralhalle 1892 , S. $61 . \ll$

1) Diese Berichte $\mathrm{XXV}, 1842$. 